\title{
The right substituent, at the right place : predicting the modulation of UV-visible absorption and emission of mono-substituted pyrido[2,3,4- $k l]$ acridines by electron density variations analysis
}

\author{
Martin Tiano*, Chloé Courdurié, Pauline Colinet \\ Laboratoire de Chimie, Université de Lyon, ENS de Lyon, CNRS UMR 5182, Université Claude Bernard Lyon 1, F69342 Lyon, France. \\ E-mail : martin.tiano@ens-lyon.fr
}

\begin{abstract}
DFT and TD-DFT calculations were performed to rationalize the relationship between UV-Visible absorption and emission spectra and the nature and position of a substituent on the heteroaromatic pyrido[2,3,4-kl]acridine skeleton. Studying the variation of electron density and partial charges between the ground state and the first excited state, we describe here a quantitative method to predict the modulation of UV-Visible spectroscopic properties depending on the nature and the position of a substituent.
\end{abstract}

Keywords: TD-DFT, Pyrido[2,3,4-kl]acridine, UV-Visible spectroscopy, Fluorescence spectroscopy, Hammett parameter

\section{Introduction}

Finding the best candidate compounds for a specific application is a long process, that usually needs to synthesize a lot of different products before identifying the good ones. For biological applications, quantitative structure-activity relationship, molecular dynamic simulation or molecular docking are rational methodologies to achieve this goal faster than blind testing.[1, 2] For spectroscopic applications in the UV-visible domain, the study of electronic transitions and the parameters which influence them could play a major role to guide the synthesis of a restricted number of candidates. In their experimental and theoretical study of Seoul-Fluor analogs, E. Kim et al. have pinpointed that the modulation of the emission wavelength of their compounds is linked to the modifications of HOMO and LUMO energies by adding diverse electron-donor or electronwithdrawing groups on one specific position.[3] Although relatively efficient in their case, this approach did not take into account the whole molecular orbitals implied in the transition between the ground state (GS) and the first excited state (ES1), nor questioned the subsitution position. Through TD-DFT calculations, we offer here to elaborate on this issue by looking at the electron density and its variation during GS-ES1 transition for different substitutions on a Pyrido[2,3,4- $k l]$ acridine nucleus. This would hopefully provide better insights and be more accurate in establishing quantitative relationship between structures and spectroscopic properties.

Pyrido[2,3,4-kl]acridine is an aromatic tetracyclic pattern which is present in almost 100 natural products isolated from sponges or tunicate species. For three decades, numerous studies showed that natural pyrido[2,3,4-kl]acridines present very interesting and various biological properties, from cytotoxicity, to antibacterial, antifungal, antiviral, insecticidal, antiHIV, and anti-parasitic activities. In particular, pyridoacridines can intercalate into DNA double-strands and interfere with topoisomerase-type enzymes. Moreover, some of them have also stronger interactions with guanine quadruplex, and inhibit telomerase complex.[4] Known pyrido[2,3,4-kl]acridines are also diversely colored and UV-visible spectra has been reported in the litterature.[5] Some of them are also fluorescent. But, to our knowledge, the only fluorescent spectrum reported to date is the one of kuanoniamine $\mathrm{B}$, a natural pentacyclic pyrido[2,3,4$\mathrm{kl}$ ] acridine $\left(\lambda_{e m}=524 \mathrm{~nm}\right.$ in methanol) [6, 7]. No other comprehensive spectroscopic study of pyrido[2,3,4- $k l]$ acridine structures has been undertaken, despite an intense and productive effort of total synthesis of the natural products members of this family.[8, 9] We think that the combination of these spectroscopic and biological properties makes pyrido[2,3,4-kl]acridine derivatives excellent candidates for bio-imaging and theranostic approaches, keeping in mind that the absorbent properties of organic tissues imply the need to tune the luminescent wavelength above $650 \mathrm{~nm}$.[10] In this work, we performed a comprehensive in silico study of UV-Visible absorption and fluorescent emission properties of mono-substituted pyridoacridines. In particular, we found that GS-ES electron density variations quantitatively correlate with the modulation of the emission and absorption wavelengths in substituted pyrido[2,3,4- $k l]$ acridine, allowing us to pre-select excellent candidates for bio-imaging applications.

\section{Methods}

\subsection{Computationnal details}

All DFT and TD-DFT calculations were performed using the Gaussian 16 program package [11]. The hybrid B3LYP $[12,13,14]$ or PBE0 [15] functionals were used together with the $6-311++G(d, p)$ basis set. Solvent effects were included either by using the conductor polarizable continuum 

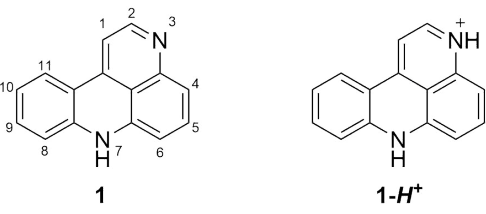

$1-H^{+}$
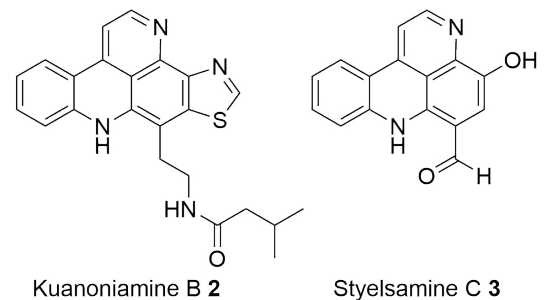

Styelsamine C 3

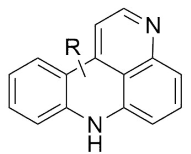

$R-1$

Styelsamine D 4

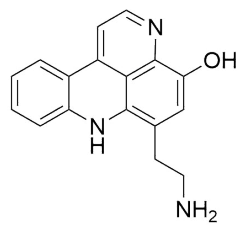

Figure 1: investigated pyrido[2,3,4- $k l]$ acridines. R represents the substituent: for example, $\mathbf{1 0 - M e - 1}$ is the pyrido[2,3,4-kl]acridine $\mathbf{1}$ with a methyl group on position 10 .

model (CPCM) 16,17$]$ and by a microsolvation appraoch using explicit solvent molecules (methanol molecules). When specified, the D3 dispersion correction proposed by Grimme et al. was used in a spirit of thoroughness.[18]

\subsection{Description/presentation of the investigated structures}

The molecules investigated in this study are pyrido[2,3,4$k l$ acridine 1, mono-substituted pyrido[2,3,4- $k l]$ acridine bearing methyl (Me) $\boldsymbol{M e} \mathbf{- 1}$, methoxy $(\mathrm{MeO}) \mathbf{M e O}-\mathbf{1}$, nitrile $(\mathrm{CN})$ $\boldsymbol{C N}$-1, formyl (Oxo) $\boldsymbol{O x o}-\mathbf{1}$, acetyl (Ac) $\boldsymbol{A c}-\mathbf{1}$, fluoro (F) $\boldsymbol{F}$ 1, trifluoromethyl $\left(\mathrm{CF}_{3}\right) \boldsymbol{C F}_{3}-\mathbf{1}$, amino $\left(\mathrm{NH}_{2}\right) \mathbf{N H}_{2}-\mathbf{1}$, methylamino (NHMe) $\mathbf{N H M e - 1}$, dimethylamino $\left(\mathrm{NMe}_{2}\right) \mathbf{N M e}-\mathbf{1}$, hydroxyl $(\mathrm{OH})$ group $\boldsymbol{O H}-1$ at diverse positions, and the following natural compounds: kuanoniamine B 2, styelsamine C 3 and D 4 (Figure 1). Since the available UV-visible spectra were realized in methanol, we used this solvent in simulations, except for $\mathbf{3}$, in chloroform.

\section{Results and discussion}

\subsection{Validation of the simulation method}

We compared the available experimental UV-visible spectra of pyrido[2,3,4- $k l]$ acridine substrates with TD-DFT calculation results. For that purpose we calculated the energy of the first electronic transition, from the ground state to the first excited state (GS-ES1 transition). In Table $1, \Delta E$ represents the difference between experimental and simulated transition energy. The best agreement between theory and experiment was obtained at B3LYP/6-311++G(d,p) level, with implicit and explicit solvation (methanol). Taking into account dispersion corrections did not decrease this gap in our case (Table 1).

These calculation were applied to other pyrido[2,3,4kl]acridine derivatives with available experimental data (Table 2). However, for Styelsamine D (4), only UV transition energies are mentioned in the litterature. So we looked directly the transition energy from ground state to the second excited state (GS-ES2). In all cases, the calculation results were in good
Table 1: Simulation parameters optimization with pyrido[2,3,4- $k l]$ acridine $\mathbf{1}$ in methanol

\begin{tabular}{llll}
\hline fonctionnal & base & solvent & $\Delta \mathrm{E}(\mathrm{eV})$ \\
\hline B3LYP & $6-31 \mathrm{G}(\mathrm{d})$ & none & 0.30 \\
B3LYP & $6-31 \mathrm{G}(\mathrm{d})$ & implicit & 0.24 \\
B3LYP & $6-31 \mathrm{G}(\mathrm{d})$ & explicit & 0.18 \\
B3LYP & $6-311++\mathrm{G}(\mathrm{d}, \mathrm{p})$ & implicit & 0.14 \\
B3LYP & $6-311++\mathrm{G}(\mathrm{d}, \mathrm{p})$ & implicit + explicit & 0.09 \\
PBE0 & $6-311++\mathrm{G}(\mathrm{d}, \mathrm{p})$ & explicit & 0.21 \\
B3LYP & $6-311++\mathrm{G}(\mathrm{d}, \mathrm{p}) *$ & implicit+explicit & 0.10 \\
*with dispersion & &
\end{tabular}

agreement with experiment, the error in energy being below $0.12 \mathrm{eV}(0.07 \mathrm{eV}$ on average). This is lower than the mean absolute error of TD-DFT calculations for singlet excited states of small organic compounds.[19].

Table 2: comparison of experimental datas and simulation (B3LYP/6$311++\mathrm{G}(\mathrm{d}, \mathrm{p})$ with implicit + explicit solvent (methanol))

\begin{tabular}{llll}
\hline Product & $\lambda_{\text {exp }}(\mathrm{nm})$ & $\lambda_{\text {sim }}(\mathrm{nm})$ & $\Delta \mathrm{E}(\mathrm{eV})$ \\
\hline $\mathbf{1}$ & $452[20]$ & 438 & 0.09 \\
$\mathbf{1 0 - M e - 1}$ & $466[20]$ & 446 & 0.12 \\
Kuanoniamine B 2 & $450[7]$ & 449 & 0.00 \\
Styelsamine C 3* & $480[21]$ & 461 & 0.11 \\
Styelsamine D 4** & $376[21]$ & 371 & 0.04
\end{tabular}

* Implicit chloroform instead of methanol.** GS-ES2 transition wavelength instead of GS-ES1 transition.

As we previously noticed, the only emission spectrum reported was that of the kuanoniamine B 2, with an maximum emission wavelength at $526 \mathrm{~nm}$. At B3LYP/6-311++G(d,p) level of theory, and explicit solvation (methanol), the simulated emission wavelength peaks at $549 \mathrm{~nm}$, that is in excellent agreement with the just mentioned experimental data $(\Delta E$ $=0.05 \mathrm{eV}) \cdot[7]$

For all further simulations, we used B3LYP/6-311++G(d,p) level of theory with implicit and explicit solvatation (methanol). We used the $t$ index defined by T. Le Bahers et al. to systematically check the accuracy of the chosen functional in the calculation of transition energy (see Supp. Info.), with respect to a problem of strong charge transfer character of a transition.[22]

\subsection{Presentation of the methodology}

We started by examining the variation of electron density between the ground state (GS) and the first excited state (ES1) of the unsubstituted pyrido[2,3,4- $k l]$ acridine to identify the spatial distribution and the amplitude of this variation, using the methodology proposed by T. Le Bahers et al. (Figure 2).[22]. On a qualitative point of view, positions 4 and 6 of the structure showed the major variation, with a decrease of the electron density. This led us to infer two hypothesis :

- Positions with the larger electron density variations are the most sensitive : substituent on that positions should greatly affect the GS-ES1 transition energy. 


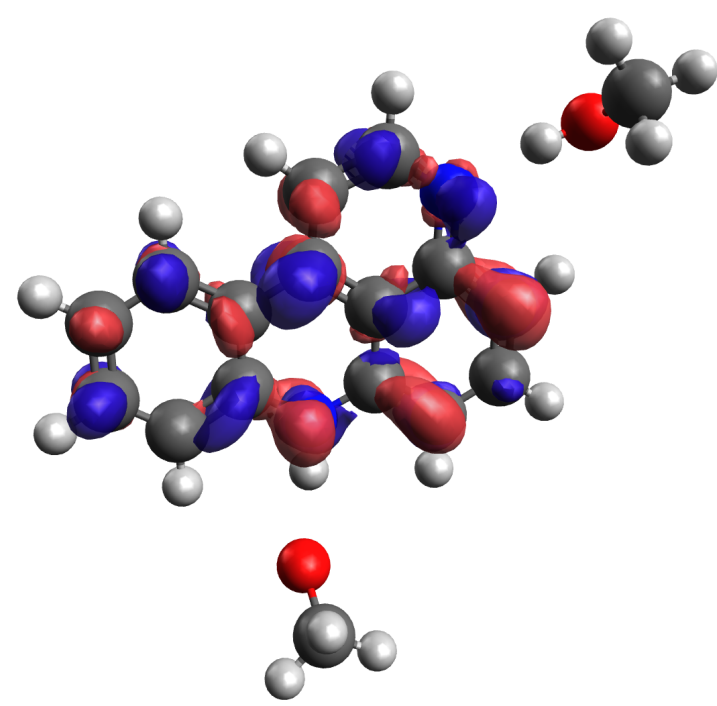

Figure 2: Difference in total electron density between ground and first excited states computed at B3LYP/6-311++G(d,p) level of theory with explicit methanol $\left(\Delta \rho(r)=\rho_{E X}(r)-\rho_{G S}(r)\right.$, isocontour value $\left.0.02 \mathrm{au}\right)$.

- For a given position, the nature of the substituent, namely how much electron donor or electron withdrawing group it is, should impact the transition (GS-ES1) energy. If the electron density variation is positive, an electron withdrawing group would stabilize the excited state, and so decrease the transition energy. On the other hand, if the electron density variation is negative, an electron withdrawing group would destabilize the excited state, and so increase the transition energy.

This behaviour would result in a wavelength shift in both absorption and emission spectra of the mono-substituted pyridoacridines compared with the unsubstituted one (1).

\subsection{The right place: Relationship between the GS-ES partial charge variations at the substituted position and absorp- tion and emission wavelengths}

We performed TD-DFT calculations of the emission energy $\Delta E_{\text {em }}$ for mono-substituted pyrido[2,3,4- $\left.k l\right]$ acridines at diverse positions with a methoxy group, and we compared these results with the $\Delta E_{e m}$ of the unsubstituted compound. The results, that are summed up in the figure 3 confirmed qualitatively the hypothesis : when a methoxy group substitutes a position where the electron density variation between GS and ES is positive, an increase of $\Delta E_{e m}$ is noticed. On the contrary, when a methoxy group substitutes a position where the electron density variation between GS and ES is negative, a decrease of $\Delta E_{e m}$ is observed. Moreover, the position 4 or 6 , where the largest absolute electron density variation between GS and ES is noticed are the most sensitive.

To perform a quantitative study of this effect, we calculated the variations of the partial atomic charges between the ground state and the first excited state (noted $\Delta \rho$ ) of each positions of the unsubstituted pyrido[2,3,4-kl]acridine, using dif-

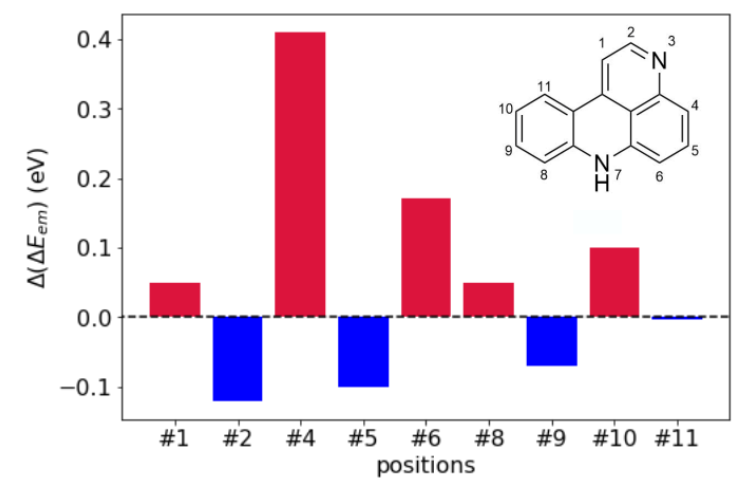

Figure 3: Amplitudes of the variation of emission energy between unsubstitued pyrido[2,3,4-kl]acridine $\mathbf{1}$ and methoxy substitued $\mathbf{1}$ in position $\#: \Delta\left(\Delta E_{e m}\right)=$ $\Delta E_{\text {em }}(\#-\mathrm{MeO}-\mathbf{1})-\Delta_{\text {em }}(\mathbf{1})$

ferent charge models (i.e. Mulliken, Merz-Kollman, Hirshfeld, CM5, Electrostatic potential charges (ESP) models).

We studied the relationship between these calculated partial charge variations and $\Delta E_{e m}$ (respectively the $\Delta E_{a b s}$ ) for monosubstituted (1) at diverse positions, using $\Delta E_{e m}(\mathbf{1})$ and $\Delta E_{a b s}(\mathbf{1})$ as references. We used the methoxy (-OMe), and the methyl groups (-Me) as models of electron donating substituent and the cyano $(-\mathrm{CN})$ group as a model of electron withdrawing substituent (Table 3).

Table 3: Correlation between $\Delta(\Delta E)=\Delta E-\Delta E(\mathbf{1})$ for $\mathrm{MeO}$ and $\mathrm{CN}$ substitued pyrido[2,3,4- $k l]$ acridine and partial charge variations between GS and ES, depending on partial charge models. In parentheses, $R^{2}$ when 5-MeO-1 or 5-CN-1 is excluded.

\begin{tabular}{llll} 
5-CN-1 is excluded. & & \\
\hline Substituent & Charge model & $\mathrm{R}^{2}(\mathrm{abs})$ & $\mathrm{R}^{2}(\mathrm{em})$ \\
\hline $\mathrm{MeO}$ & Mulliken & $0.78(0.79)$ & $0.84(0.85)$ \\
$\mathrm{MeO}$ & Merz-Kollman & $0.91(0.90)$ & $0.90(0.89)$ \\
$\mathrm{MeO}$ & Hirshfeld, CM5 & $0.80(0.93)$ & $0.82(0.94)$ \\
$\mathrm{MeO}$ & ESP & $0.42(0.76)$ & $0.52(0.89)$ \\
$\mathrm{CN}$ & Mulliken & $0.64(0.77)$ & $0.56(0.77)$ \\
$\mathrm{CN}$ & Merz-Kollman & $0.64(0.94)$ & $0.54(0.96)$ \\
$\mathrm{CN}$ & Hirshfeld, CM5 & $0.92(0.95)$ & $0.88(0.96)$ \\
$\mathrm{CN}$ & ESP & $0.86(0.86)$ & $0.88(0.86)$ \\
$\mathrm{Me}$ & Mulliken & $0.55(0.54)$ & $0.51(0.52)$ \\
$\mathrm{Me}$ & Merz-Kollman & $0.59(0.58)$ & $0.50(0.57)$ \\
$\mathrm{Me}$ & Hirshfeld, CM5 & $0.60(0.64)$ & $0.60(0.67)$ \\
$\mathrm{Me}$ & ESP & $0.29(0.44)$ & $0.46(0.59)$ \\
\hline
\end{tabular}

Merz-Kollman, and Hirshfeld and CM5 models gave the strongest correlations for both methoxy and cyano substituted structures (Figures 4 and 5). In the case of methyl substituted pyridoacridines, weak correlations are observed, due to the very small energy variation between the substituted and the unsubstituted pyrido[2,3,4- $k l]$ acridines. We noticed that calculated energies for 5-MeO-1 and 5-CN-1 do not fit as well as the other positions, especially for 5-CN-1 with Merz-Kollman charge model and for 5-MeO-1 with Hirshfeld and CM5 models. The special behavior of that structures could be explained by the relatively small charge variations which occur at position 5 , and by the fact that sign of atomic partial charge vari- 


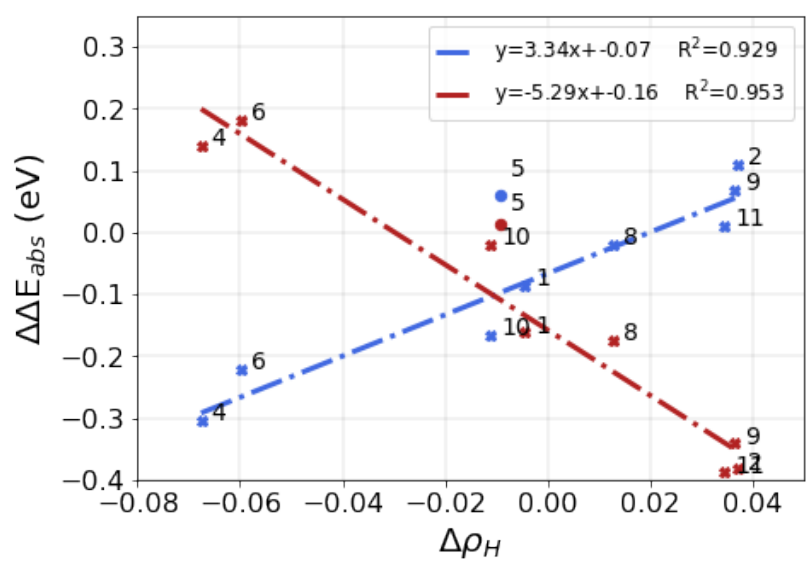

Figure 4: Correlation between $\Delta\left(\Delta E_{a b s}\right)=\Delta E_{a b s}$ (\#-MeO-1)- $\Delta E_{a b s}(\mathbf{1})$ (blue line) and $\Delta\left(\Delta E_{a b s}\right)=\Delta E_{a b s}$ (\#-CN-1)- $\Delta E_{a b s}(\mathbf{1})$ (red line) and the partial charge variations between GS and ES of the carbon in position \# in compound 1 (Hirshfeld charge model). Line equation and $\mathrm{R}^{2}$ calculated without position 5 .

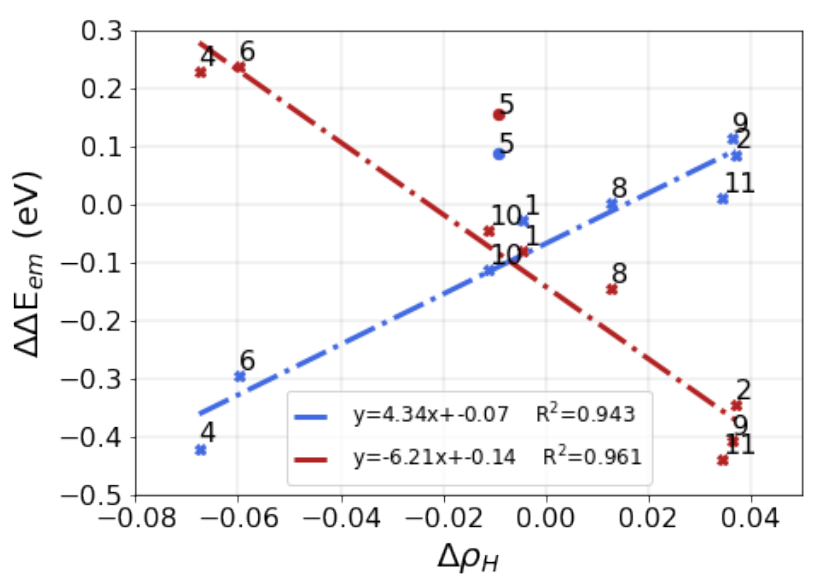

Figure 5: Correlation between $\Delta\left(\Delta E_{\text {em }}\right)=\Delta E_{\text {em }}$ (X-MeO-1) $-\Delta E_{\text {em }}$ (1) (blue line) and $\Delta\left(\Delta E_{\text {em }}\right)=\Delta E_{\text {em }}(\mathbf{X}-\mathbf{C N}-\mathbf{1})-\Delta E_{\text {em }}(\mathbf{1})$ (red line) and the partial charge variations between GS and ES of the carbon in position \# in compound $\mathbf{1}$ (Hirshfeld charge model). \# is the position of Methoxy (blue line) and Cyano (red line) substituents.(Line equation and $\mathrm{R}^{2}$ calculated without position 5)

ations in that position differs from Merz-Kollman to Hirshfeld or CM5 models (see Supp. Info.). It is noteworthy that in a TD-DFT study of charge-transfer excitations, D. Jacquemin et al. showed that the partitioning schemes fitting the electrostatic potential, as Merz-Kollman model, at the contrary of Hirshfeld model, were the most consistent for the simulation of the reorganisation of the electronic cloud during excitation.[23] In our case, where charge transfers are significantly lower, Hirshfeld or CM5 models gave the best correlations (with or without considering the position 5 substituted molecules), showing that atomic partial charge model has to be carefully chosen to be consistent with the studied phenomenon.

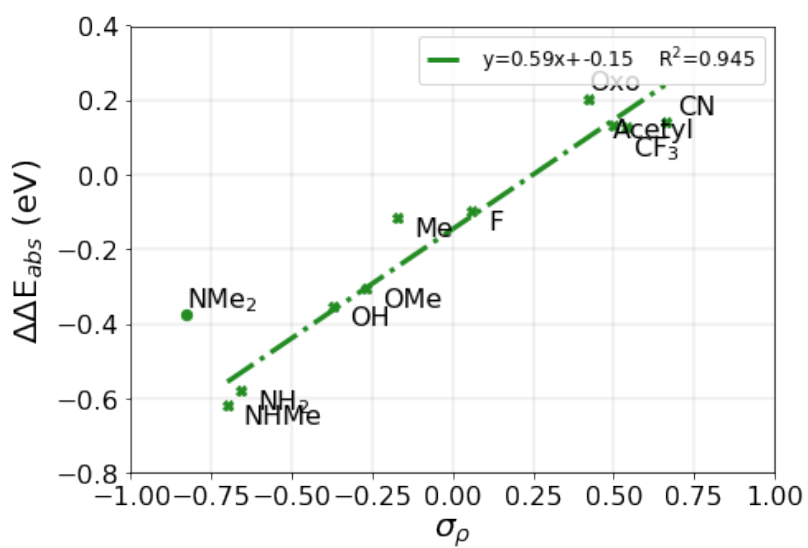

Figure 6: Correlation between $\Delta\left(\Delta E_{a b s}\right)=\Delta E_{a b s}(\mathbf{4 - \# - 1})-\Delta E_{a b s}(\mathbf{1})$ and the Hammett Parameter $\sigma_{p}$ of the substituent \#.

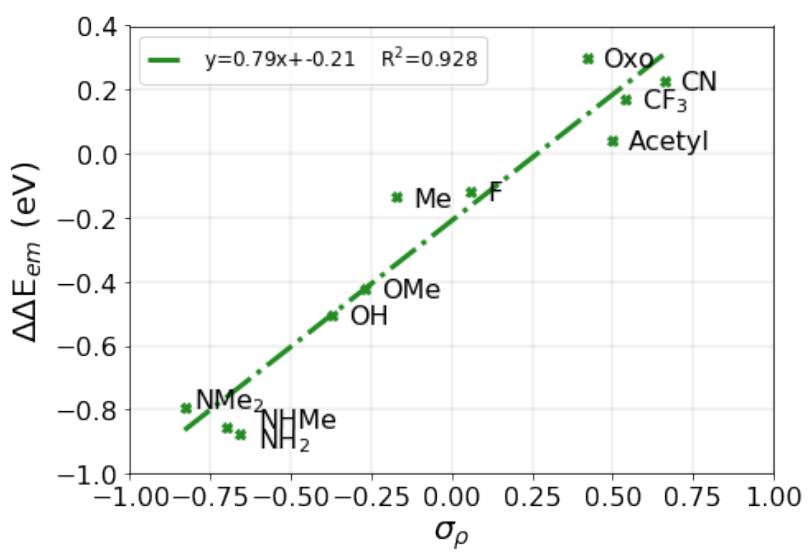

Figure 7: Correlation between $\Delta\left(\Delta E_{e m}\right)=\Delta E_{e m}(\mathbf{4 - \# - 1})-\Delta E_{a b s}(\mathbf{1})$ and the Hammett Parameter $\sigma_{p}$ of the substituent \#.

\subsection{The right substituent : Relation between Hammett param- eter of the substituent and absorption and emission wave- length}

We focused on the more sensitive positions (i.e. positions 4,6 , and 2), and calculate $\Delta E_{e m}$ and $\Delta E_{a b s}$ for different electron donor (EDG) or electron withdrawing groups (EWG). Confirming our intuition and our first results (see section 3.3), the substitution of the positions 4 or 6 with an EWG (respectively an EDG) implies an increase (respectively a decrease) of both $\Delta E_{e m}$ and $\Delta E_{a b s}$, and the opposite results were obtained with position 2 .

To quantify this effect, we used $\sigma_{p}$ and $\sigma_{m}$ Hammett parameters and the Remya parameter $\Delta V_{C}$, as numeric scales for the electron withdrawing or the electron donating strength of the substituents, $[24,25,26]$. We calculated $\Delta\left(\Delta E_{e m}\right)$ and $\Delta\left(\Delta E_{a b s}\right)$ for mono-substituted pyrido[2,3,4-kl] acridines in position $2(9$ examples), 4 (11 examples) and 6 (10 examples). The best correlations were obtained with $\sigma_{p}$ and $\Delta V_{C}$ parameters (Figures 6 and 7, and Supp. Info.).

For positions 4 and $6, \Delta\left(\Delta E_{a b s}\right)$ and $\Delta\left(\Delta E_{e m}\right)$ clearly correlated linearly with $\sigma_{p}$ and $\Delta V_{C}$. However, we noticed that the 
calculated $\Delta\left(\Delta E_{a b s}\right)$ of $4-N M e_{2}$ and $\mathbf{6}-N M e_{2}$ substituted structures did not fit correctly, though $\Delta\left(\Delta E_{e m}\right)$ did.

In these cases, $\Delta\left(\Delta E_{a b s}\right)$ were notably smaller than our model predictions. Actually, due to steric constaints, the nitrogen lone pair of $\mathrm{NMe}_{2}$ is not aligned with the $\pi$ system of the pyridoacridine core. This geometrical distortion of the $\pi$ system probably leads to an increase of the GS energy, and explains why $\sigma_{p}$ and $\Delta V_{C}$ failed to reflect the electron-donating strength of $\mathrm{NMe}_{2}$ substituent. Nevertheless, the relaxed geometry at the excited state presents a $15^{\circ}$ rotation of the bond between $\mathrm{NMe}_{2}$ and the pyridoacridine core, with a better alignment of the nitrogen lone pair with the $\pi$ system. In that geometry, $\sigma_{p}$ and $\Delta V_{C}$ reflect more the electron-donating strength of $\mathrm{NMe}_{2}$ substituent, that explains the accuracy of our model for $\Delta\left(\Delta E_{e m}\right)$ calculations. (see Supp. Info.) Interestingly, when that substituent is excluded, $R^{2} \simeq 0,92$ for all calculations with $\sigma_{p}$ parameter. The correlations were however weaker $\left(R^{2} \simeq 0,6-0,7\right)$ for substitutions in position 2 . The quite large variations of the transition energy from one to another substituent on that position probably reflect the strong influence of the nitrogen lone pair in position 3.

In a study about substituted aryl hydrazones of 1,8naphthalimide, D. Cheshmedzhieva et al. have already highlighted correlations between $\sigma_{p}$ and $\lambda_{\text {fluo }}$ for 5 monosubstituted structures, but they noticed that correlation differs in regard to the ED or EW status of the substituent, that they interpreted as a consequence of large charge transfers.[27] In our case, it is noteworthy that calculated charge transfers are modest for the studied mono-substituted pyrido[2,3,4-kl]acridines, as evidenced by the evaluation of the parameters $\mathrm{D}_{C T}$ (CT distance), and $\mu_{C T}$ (see Supp. Info.). Thus, as expected, we did not observe any slope breaks between EDG and EWG correlations with $\sigma_{p}$ nor $\Delta V_{C}$ in our cases.

\section{Conclusion}

In our attempt to rationalize the effort to tune the UV-Visible spectroscopic properties of pyrido[2,3,4-kl]acridine derivatives, we investigated a simple in silico methodology to predict the influence of both the position and the nature of a substituent on the lowest absorption energy and emission energy. Except for one position, the variation of partial charges between the GS and the ES1 of the unsubstitued pyrido[2,3,4-k1]acridine calculated by (TD)-DFT with Hirshfeld, CM5 or Merz-Kollman models accurately reflected the sensitivity of each positions, i.e. how much a given substituent on the position change the absorption or emission transition energy. In a second aspect, for a given position on the aromatic core, we showed that the variations of $\Delta E_{e m}$ and $\Delta E_{a b s}$ ) between the mono-substitued structure and the unsubstituted one are quantitatively linked to the Hammett parameter $\sigma_{p}$ and Remya parameter $\Delta V_{C}$. Thanks to this work, we have identified several candidates with probable near infrared fluorescence, the synthesis of which are currently underway. We believe that this methodology should be successfully applied on other fluorescent structures, and should save time and energy to find the best adapted candidates for nu- merous applications, from bio-imaging to organic luminescent devices.

\section{Author contributions}

Martin Tiano : Conceptualization, Methodology, Supervision, Investigation, Writing -Original draft- and -Review and editing-.

Chloé Courdurié : Investigation, Writing -Review and editing-, Visualization

Pauline Colinet : Investigation, Validation, Methodology, Visualization, Writing -Original draft- and -Review and editing-.

\section{Conflict of Interest}

The authors declare no competing financial interest.

\section{Aknowledgement}

The authors acknowledge the SYSPROD project and AXELERA Pôle de Compétitivité for financial support (PSMN Data Center) and Dr. Tangui Le Bahers for very useful discussions.

\section{References}

[1] G. R. Sliwoski, J. Meiler, E. W. Lowe, Computational Methods in Drug Discovery, Pharmacological Reviews 66 (1) (2014) 334-95. doi:10.1124/pr.112.007336.

[2] D. Ramírez, Computational Methods Applied to Rational Drug Design, The Open Medicinal Chemistry Journal 10 (1) (2016) 7-20. doi:10.2174/1874104501610010007.

[3] E. Kim, Y. Lee, S. Lee, S. B. Park, Discovery, Understanding, and Bioapplication of Organic Fluorophore: A Case Study with an Indolizine-Based Novel Fluorophore, Seoul-Fluor, Accounts of Chemical Research 48 (3) (2015) 538-547. doi:10.1021/ar500370v.

[4] S. R. Ibrahim, G. A. Mohamed, Marine Pyridoacridine Alkaloids: Biosynthesis and Biological Activities, Chemistry and Biodiversity 13 (1) (2016) 37-47. doi:10.1002/cbdv.201400434.

[5] S. R. Ibrahim, G. A. Mohamed, Pyridoacridine alkaloids from deep-water marine organisms: Structural elucidation, Bulletin of Faculty of Pharmacy, Cairo University 54 (2) (2016) 107-135. doi:10.1016/j.bfopcu.2016.08.003.

[6] A. R. Carroll, P. J. Scheuer, Kuanoniamines A, B, C, and D: Pentacyclic Alkaloids from a Tunicate and Its Prosobranch Mollusk Predator Chelynotus semperi, Journal of Organic Chemistry 55 (14) (1990) 4426-4431. doi:10.1021/jo00301a040.

[7] G. P. Gunawardana, F. E. Koehn, A. Y. Lee, J. Clardy, H. yin He, D. J. Faulkner, Pyridoacridine Alkaloids from Deep-Water Marine Sponges of the Family Pachastrellidae: Structure Revision of Dercitin and Related Compounds and Correlation with the Kuanoniamines, Journal of Organic Chemistry 57 (5) (1992) 1523-1526. doi:10.1021/jo00031a035.

[8] D. Skyler, C. H. Heathcock, The pyridoacridine family tree: A useful scheme for designing synthesis and predicting undiscovered natural products, Journal of Natural Products 65 (11) (2002) 1573-1581. doi:10.1021/np020016y.

[9] J. A. Joule, M. Álvarez, Pyridoacridines in the 21st Century, European Journal of Organic Chemistry 2019 (31-32) (2019) 5043-5072. doi:10.1002/ejoc.201900401.

[10] J. B. Li, H. W. Liu, T. Fu, R. Wang, X. B. Zhang, W. Tan, Recent Progress in Small-Molecule Near-IR Probes for Bioimaging, Trends in Chemistry 1 (2) (2019) 224-234. doi:10.1016/j.trechm.2019.03.002. 
[11] M. J. Frisch, G. W. Trucks, H. B. Schlegel, G. E. Scuseria, M. A. Robb, J. R. Cheeseman, G. Scalmani, V. Barone, G. A. Petersson, H. Nakatsuji, X. Li, M. Caricato, A. V. Marenich, J. Bloino, B. G. Janesko, R. Gomperts, B. Mennucci, H. P. Hratchian, J. V. Ortiz, A. F. Izmaylov, J. L. Sonnenberg, D. Williams-Young, F. Ding, F. Lipparini, F. Egidi, J. Goings, B. Peng, A. Petrone, T. Henderson, D. Ranasinghe, V. G. Zakrzewski, J. Gao, N. Rega, G. Zheng, W. Liang, M. Hada, M. Ehara, K. Toyota, R. Fukuda, J. Hasegawa, M. Ishida, T. Nakajima, Y. Honda, O. Kitao, H. Nakai, T. Vreven, K. Throssell, J. A. Montgomery, Jr., J. E. Peralta, F. Ogliaro, M. J. Bearpark, J. J. Heyd, E. N. Brothers, K. N. Kudin, V. N. Staroverov, T. A. Keith, R. Kobayashi, J. Normand, K. Raghavachari, A. P. Rendell, J. C. Burant, S. S. Iyengar, J. Tomasi, M. Cossi, J. M. Millam, M. Klene, C. Adamo, R. Cammi, J. W. Ochterski, R. L. Martin, K. Morokuma, O. Farkas, J. B. Foresman, D. J. Fox, Gaussian 16 Revision C.01, gaussian Inc. Wallingford CT (2016).

[12] A. D. Becke, A new mixing of Hartree-Fock and local density-functional theories, The Journal of Chemical Physics 98 (2) (1993) 1372-1377. doi:10.1063/1.464304.

[13] C. Lee, C. Hill, N. Carolina, Development of the Colle-Salvetti correlation-energy formula into a functional of the electron density, Chemical Physics Letters 162 (3) (1989) 165-169. arXiv:PhysRevA.38.3098, doi:10.1016/0009-2614(89)85118-8.

[14] S. H. Vosko, L. Wilk, M. Nusair, Accurate spin-dependent electron liquid correlation energies for local spin density calculations: a critical analysis, Canadian Journal of Physics 58 (8) (1980) 1200-1211. doi:10.1139/p80159.

[15] C. Adamo, V. Barone, Toward reliable density functional methods without adjustable parameters: The PBE0 model, Journal of Chemical Physics 110 (13) (1999) 6158-6170. doi:10.1063/1.478522.

[16] V. Barone, M. Cossi, Quantum calculation of molecular energies and energy gradients in solution by a conductor solvent model, Journal of Physical Chemistry A 102 (11) (1998) 1995-2001. doi:10.1021/jp9716997.

[17] M. Cossi, N. Rega, G. Scalmani, V. Barone, Energies, structures, and electronic properties of molecules in solution with the C-PCM solvation model, Journal of Computational Chemistry 24 (6) (2003) 669-681. doi: $10.1002 / j$ jcc. 10189 .

[18] S. Grimme, S. Ehrlich, L. Goerigk, Effect of the damping function in dispersion corrected density functional theory, Journal of Computational Chemistry 32 (7) (2011) 1456-1465. doi:https://doi.org/10.1002/jcc.21759.

[19] D. Jacquemin, V. Wathelet, E. A. Perpète, C. Adamo, Extensive TDDFT benchmark: Singlet-excited states of organic molecules, Journal of Chemical Theory and Computation 5 (9) (2009) 2420-2435. doi: $10.1021 / \mathrm{ct} 900298 \mathrm{e}$.

[20] G. Gellerman, A. Rudi, Y. Kashman, Synthesis of pyrido[2,3,4$\mathrm{kl}$ ]acridines a building block for the synthesis of pyridoacridine alkaloids, Tetrahedron Letters 33 (38) (1992) 5577-5580. doi:10.1016/S00404039(00)61150-4.

[21] B. R. Copp, J. Jompa, A. Tahir, C. M. Ireland, Styelsamines A-D: New tetracyclic pyridoacridine alkaloids from the indonesian ascidian eusynstyela latericius, Journal of Organic Chemistry 63 (22) (1998) 8024-8026. doi:10.1021/jo980905j.

[22] T. Le Bahers, C. Adamo, I. Ciofini, A qualitative index of spatial extent in charge-transfer excitations, Journal of Chemical Theory and Computation 7 (8) (2011) 2498-2506. doi:10.1021/ct200308m.

[23] D. Jacquemin, T. L. Bahers, C. Adamo, I. Ciofini, What is the "best" atomic charge model to describe through-space charge-transfer excitations?, Physical Chemistry Chemical Physics 14 (16) (2012) 5383-5388. doi: $10.1039 / \mathrm{c} 2 \mathrm{cp} 40261 \mathrm{k}$.

[24] L. P. Hammett, The Effect of Structure upon the Reactions of Organic Compounds. Benzene Derivatives, Journal of the American Chemical Society 59 (1) (1937) 96-103. doi:10.1021/ja01280a022.

[25] C. Hansch, A. Leo, R. W. Taft, A Survey of Hammett Substituent Constants and Resonance and Field Parameters, Chemical Reviews 91 (2) (1991) 165-195. doi:10.1021/cr00002a004.

[26] G. S. Remya, C. H. Suresh, Quantification and classification of substituent effects in organic chemistry: A theoretical molecular electrostatic potential study, Physical Chemistry Chemical Physics 18 (30) (2016) 2061520626. doi:10.1039/c6cp02936a.

[27] D. Cheshmedzhieva, P. Ivanova, S. Stoyanov, D. Tasheva, M. Dimitrova, I. Ivanov, S. Ilieva, Experimental and theoretical study on the absorp- tion and fluorescence properties of substituted aryl hydrazones of 1,8naphthalimide, Physical Chemistry Chemical Physics 13 (41) (2011) 18530-18538. doi:10.1039/c1cp21756a. 